\title{
UMA DISCUSSÃO FILOSÓFICA DOS MÉTODOS DE AVALIAÇÃO DO NÍVEL DE CONSCIÊNCIA
}

A PHILOSOPHICAL DISCUSSION OF THE METHODS OF EVALUATION OF THE

LEVEL OF CONSCIOUSNESS

\author{
MARCELO AlEXANDRE AlBiNo FilHO ${ }^{1}$ \\ Universidade do Oeste Paulista (UNOESTE) - Brasil \\ marceloalexandrealbino@hotmail.com
}

ALFREDO PEREIRA JUNIOR ${ }^{2}$

Universidade Estadual Paulista (UNESP) - Brasil

alfredo.pereira@gmail.com

RESUMO: Nas últimas décadas, a temática da consciência tem atraído atenção de pesquisadores em Filosofia da Mente, e em diversas disciplinas científicas e tecnológicas, inclusive na área de Saúde. O objetivo deste artigo é identificar uma possível relação entre métodos quantitativos de avaliação dos estados de consciência e os aspectos subjetivos da experiência em primeira pessoa, deste modo evitando uma postura cética a respeito do "Problema Difícil da Consciência" formulado por David Chalmers. No Monismo Dual, formulado por Max Velmans, a mensuração da atividade cerebral e do comportamento (incluindo a quantificação de categorias em relatos da experiência subjetiva), realizada na perspectiva de terceira pessoa (cientista), têm como referente os mesmos processos que geram a experiência subjetiva (qualidades perceptivas e sentimentos vivenciados pela pessoa atendida), na perspectiva de primeira pessoa. Portanto, encontramos nesta abordagem filosófica uma justificativa epistemológica para a correspondência entre os dados obtidos em medidas objetivas e o estado consciente subjetivo experimentado pela pessoa atendida no sistema de saúde.

PALAVRAS-CHAVE: Nível de Consciência. Escalas. Conceito de Consciência. Avaliação Psicológica. Filosofia da Mente.

ABSTRACT: In the last decades, the theme of consciousness has attracted the attention of researchers in Philosophy of Mind, and in several scientific and technological disciplines, including in the area of Health. The objective of this article is to identify a possible relation between quantitative methods of evaluation of states of consciousness and the subjective aspects of firstperson experience, thus avoiding a skeptical stance on the "Hard Problem of Consciousness" as formulated by David Chalmers. In the philosophy of Dual Monism, formulated by Max Velmans, the measurement of brain activity and behavior (including reports of subjective experience), carried out from the perspective of the third person (scientist), refer to the same processes that generate subjective experience (qualities and feelings of the attended person) from the first person perspective. Therefore, we find in this philosophical approach an epistemological justification for the correspondence between the data obtained in objective measures and the subjective conscious state experienced by the person served in the health system.

KEYWORDS: Level of Consciousness. Scales. Concept of Consciousness. Psychological Evaluation. Philosophy of Mind.

\footnotetext{
${ }^{1}$ Mestre em Filosofia e professor na Universidade do Oeste Paulista (UNOESTE).

${ }^{2}$ Livre-Docente, professor da Universidade Estadual Paulista Júlio de Mesquita Filho (UNESP).
} 


\section{INIRODUÇ̃̃O}

Este artigo tem por objetivo identificar uma possível relação entre métodos quantitativos de avaliação dos estados de consciência e os aspectos subjetivos da experiência em primeira pessoa. Para isto, depara-se com o que Chalmers (1996) denominou de problemas do estudo da consciência: "problemas fáceis" e "problema difícil". Os problemas fáceis dizem respeito ao estudo de fenômenos observáveis relacionados à consciência, na neurociência, na psicologia comportamental e psicofísica. Já o problema difícil consiste em explicar porquê o desempenho das funções fisiológicas e mentais (cognitivas, afetivas e comportamentais) é acompanhando por uma experiência consciente.

Para Chalmers (1996) há uma "lacuna explicativa" entre os aspectos funcionais e os fenômenos da consciência. Assim, adota uma posição monista de substância a respeito dos sistemas conscientes, ou seja, considera mente e corpo como compondo um único sistema, ao mesmo tempo em que adota o dualismo de propriedades (Chalmers, 1996), uma posição que considera as propriedades da experiência consciente como pertencendo a um domínio disjunto das propriedades dos processos físicos, químicos, biológicos e computacionais que suportam as funções mentais.

Nos cenários da saúde, a avaliação dos estados de consciência é prática essencial, pois é a partir dela que condutas terapêuticas são tomadas. Há várias modalidades e técnicas de avaliação do nível de consciência, por meio de mensuração da atividade cerebral, de sinais comportamentais e de análise de relatos da experiência consciente pela pessoa atendida. Neste artigo analisamos alguns destes critérios vigentes na prática da saúde para a avaliação da consciência, discutindo o conceito de nível de consciência e as condições necessárias para a ocorrência da experiência consciente, a fim de estabelecer uma possível relação entre os aspectos objetivos e subjetivos da experiência consciente.

Para isto, utiliza-se como referencial teórico o livro Understanding Consciousness de Max Velmans (2009), psicólogo britânico que utiliza a fenomenologia para compreensão dos estados da consciência. A partir desse referencial, analisa-se os critérios utilizados na prática clínica para se aferir a presença ou ausência da experiência consciente, e o uso de escalas unidimensionais e multidimensionais, como tentativa de quantificar o nível de experiência consciente.

\section{MONISMO}

Velmans (2009, p. 132) considera que o fenômeno consciência têm dois aspectos: um deles abordável cientificamente, na perspectiva da terceira pessoa, e o outro restrito à experiência individual, na perspectiva de primeira pessoa. Como os dois aspectos dizem respeito a um mesmo sistema, é possível supor que corpo e mente são aspectos do mesmo sistema e, conseqüentemente, haveria uma correlação entre o nível de consciência de uma pessoa e os sinais objetivos que seu corpo expressa, que são mensuráveis por um observador externo. Embora não 
se proponha a identidade entre estados corporais e estados mentais conscientes, o Monismo implica na existência de um mesmo sistema gerador de ambos, o que desautoriza o dualismo de propriedades, de Chalmers, em prol de uma conaturalidade de corpo e mente.

Desta maneira, haveria uma co-originação dos fenômenos corporais e mentais de uma pessoa, tendo a informação como mediadora. Padrões de informação processados inconscientemente pelo sistema nervoso, quando atingem determinados limiares, se tornam conscientes para a pessoa portadora deste sistema nervoso. Para uma terceira pessoa, estes padrões de informação podem ser registrados, analisados e correlacionados com estados mentais conscientes (VELMANS, 2009, p. 267).

Este raciocínio nos conduz à questão seguinte: como os padrões de informação processados pelo sistema nervoso tornam-se conscientes? Velmans (2009, p. 128) enfocou, na formulação de sua teoria, o caso de estímulos sensoriais que geram experiências conscientes localizadas no espaço-tempo fenomenal. Entretanto, na avaliação dos níveis de consciência, nem sempre se pode reduzir a existência da consciência à resposta a estímulos externos, envolvendo processamento de informação no neocortex. Os aspetos neuroanatômicos e neurofuncionais básicos da consciência estão relacionados ao processamento realizado nos núcleos intralaminares do tronco cerebral e na formação reticular (KANDEL et al, 2014; MACHADO, 2007).

\section{NÍVEL DE CONSCIÊNCIA}

Entende-se por nível de consciência a variação do estado consciente da pessoa em relação ao mundo externo, indo da excitação e vigilância à sonolência e torpor. Destaca-se o papel da formação reticular, pois envolve múltiplos neurotransmissores e conexões com áreas importantes do sistema nervoso central (KANDEL et al, 2014). Além disso, Schurger et al (2010) indicam que além das estruturas cerebrais importantes para o processamento consciente, o padrão temporal de atividade neuronal também é significativo, pois a capacidade de reproduzi-lo com similaridade em diferentes instâncias da mesma percepção indica atividade consciente.

$\mathrm{Na}$ abordagem monista, a experiência consciente não se limita ao aspecto fisiológico do sistema nervoso, mas se considera que o sistema nervoso também tenha, intrinsecamente, aspectos informacionais e sentientes (Pereira Jr., 2014). Como argumenta Chomsky (2006), o termo "físico" não tem significado filosófico preciso, podendo se restringir àquilo que é puramente material, no sentido da física clássica (aquilo que tem massa e volume), ou se estender para o domínio total dos processos estudados pela ciência física, que inclui conceitos próximos do domínio psicológico, como por exemplo os conceitos de superposição e emaranhado quânticos.

Com base no Monismo Dual, argumentamos que, na prática clínica, haveriam correspondências entre as atividades fisiológicas, informacionais e 
sentientes intrínsecas aos sistemas nervosos dos seres vivos, possibilitando que indicadores objetivos possam ser utilizados para se avaliar o nível de consciência. Para Velmans (2009, p. 313): "A consciência e seus correlatos físicos são, na verdade, aspectos complementares de uma mente psicofísica, onde podemos unificar a consciência ao cérebro, preservando o estatuto ontológico de ambos".

Como exemplo, podemos citar a ação de drogas supressoras da consciência; as mais utilizadas nos cenários da saúde, em especial, em procedimentos anestésicos, suprimem a atividade consciente de modo regular e previsível. Durante a anestesia geral, os fármacos induzem um estado reversível de amnésia, imobilidade, atenuação das respostas autônomas, analgesia e inconsciência (EVERS, CROWDER, 2005). Neste sentido, Fagundes (2018, p. 194) argumenta que a subjetividade presente nos diversos estados mentais é constituída a partir das relações que o sujeito possui com os outros e com o mundo. Logo, é possível inferir o estado da consciência do indivíduo por meio do discurso próprio do sujeito acerca de seus estados mentais subjetivos.

Coleman (2019) argumenta que as características subjetivas e objetivas da experiência consciente estão em uma relação intencional que sustenta intimidade fenomenológica. Exemplificam a dor como uma experiência sensorial e o sentimento de dor que pode acompanhá-la como fenômenos inseparáveis. Neste sentido, "não há nenhum tipo de estado mental isolado, pois atitudes proposicionais fazem parte de uma rede de relações com nossas sensações e comportamentos, além de estarem conectadas também ao mundo externo" (FAGUNDES, 2018, p. 190).

O Monismo aqui assumido permite inferir que os estados da consciência possuem componentes objetivos e subjetivos que são inseparáveis e ontologicamente intrínsecos aos seres vivos. Desta forma, é possível discutir os sinais objetivos da experiência consciente, a fim de diminuir o problema de assimetria entre os aspectos epistemológicos de primeira e terceira pessoa quando associados à prática clínica.

\section{AVALIAÇÃO DO NÍVEL DE CONSCIÊNCIA}

As escalas de avaliação do nível de consciência utilizadas no cenário da saúde consideram sinais objetivos apresentados pelos indivíduos como indicadores de ocorrência da experiência consciente. Desta forma, medidas da atividade cerebral e sinais comportamentais são utilizados como critérios de avaliação. Entende-se que os sinais objetivos da consciência (abertura ocular, respostas verbal, motora e dolorosa) são processos cerebrais aos quais possivelmente corresponde a uma atividade consciente.

A dificuldade é mais aguda quando os sujeitos se encontram anestesiados ou com prejuízo das funções motoras, necessitando, assim, de exames complementares para melhor respaldo na avaliação, como o uso do eletroencefalograma (EEG) e do índice BIS (JHON et al., 2011). 
O eletroencefalograma (EEG) é o registro da atividade elétrica espontânea do cérebro resultante dos potenciais inibitórios e excitatórios pós-sinápticos do córtex cerebral. Os sinais elétricos são registrados como ondas de voltagem em função do tempo. Já o índice BIS, por sua vez, é derivado do EEG que mede as relações entre os componentes sinusoidais do EEG (frequência, fase e amplitude) por meio de um cálculo estatístico (DUARTE, SARAIVA, 2009; PEDROZA, 2011).

Por meio das escalas de avaliação do nível de consciência é possível identificar que os sinais objetivos da consciência são classificados em abertura ocular, respostas verbal, motora e dolorosa. Tais parâmetros parecem ser importantes para inferir o nível de consciência do indivíduo, pois são estes que são avaliados nas principais escalas utilizadas no cenário da saúde. São elas: Escala de Coma de Glasgow (1976), com revisão em 1985 e mais atualmente em 2018; Escala de Coma de Jouvet (1969); Reaction Level Scale (1985); Sistema de Pontuação FOUR (2005); Índice de Aldret-Kroulik (1970); Escala de Ramsay (1974); Escore de Richmond (MENDES et al, 2008; CASTRO et al, 2012; OLIVEIRA, PEREIRA, FREITAS, 2014; BORDINI et al, 2010).

O avaliador utiliza os sinais objetivos da consciência apresentados pelo indivíduo para inferir o nível de consciência deste. Metodologicamente, cada escala possui um escore de pontuação e a cada resposta emitida pelo indivíduo frente o estímulo evocado pelo avaliador, atribui-se uma nota numérica. O resultado final é classificado dentro dos parâmetros de alerta/acordado; sonolento/letárgico; torporoso; e comatoso (NITRINI, 2010).

A inferência de que a abertura ocular e as respostas verbal e dolorosa são sinais objetivos da experiência consciente só é possível quando considera-se que mente e corpo são aspectos da mesma unidade funcional, pois parte-se do pressuposto de que há correlação da unidade dos aspectos: considerando-se os correlatos neurais da consciência, para cada processo físico-biológico descritível na perspectiva de terceira pessoa corresponde necessariamente a uma experiência consciente na perspectiva de primeira pessoa.

A resposta verbal, na maioria das vezes, pode estar acompanhada de consciência. Assim, é um parâmetro confiável para ocorrência da experiência consciente, pois a capacidade do indivíduo em expressar seus pensamentos por meio da fala, diz respeito à experiência subjetiva, e como esta pertence a mesma unidade funcional, pode-se inferir o nível de consciência. Entretanto, a experiência consciente pode ocorrer sem necessariamente a presença da fala do indivíduo, sendo esta apenas uma forma de acesso à experiência subjetiva.

Desta maneira, a resposta verbal é um indicador importante para compreender a experiência subjetiva do indivíduo, porém a consciência pode ocorrer sem a obrigatoriedade deste aspecto. LeDoux (2003) argumenta em seus trabalhos sobre o medo que a fala do indivíduo pode confundir o avaliador, pois é mais um elemento a ser avaliado e não, necessariamente, a ocorrência da experiência consciente.

Entretanto, na área da saúde, este aspecto é bastante importante para inferir o nível de consciência, pois o relato verbal é de fácil acesso pelo avaliador e 
corresponde ao que Velmans (2009) afirma ser necessário em toda avaliação, a intersubjetividade como um acordo coletivo para que as condições de observações sejam suficientemente normatizadas dentro da comunidade científica, ou seja, toda avaliação possui seu caráter subjetivo, e este manifesta-se pela relação dos diversos estados mentais; portanto, a interpretação dada pelo avaliador tem grandes chances de estar correta (FAGUNDES, 2018).

A resposta motora voluntária é consciente. Quando um indivíduo sofre bloqueio anestésico em algum plexo nervoso, não deixa de estar consciente de si e do mundo fenomenal. Assim, por meio do Monismo Dual e de seu desenvolvimento em uma Teoria Projetiva da Consciência (Pereira Jr., 2018), é possível compreender que, nesta situação, a projeção dos conteúdos da consciência para o mundo fenomenal ocorrem normalmente, corroborando, mais uma vez, para a unidade dos aspectos objetivos e subjetivos.

Além disso, é importante citar que os parâmetros motores involuntários não são dados observáveis de terceira pessoa confiáveis para avaliação do nível de consciência, pois, basicamente, se resumem a reflexos medulares, não atingindo o sistema nervoso central e, por consequência, os limiares necessários para ocorrência da experiência consciente.

A resposta dolorosa também é um importante indicador da experiência consciente. Devido a sua grande complexidade, a avaliação e mensuração da dor é tema importante nos cenários da saúde e filosófico. Parte-se do pressuposto de que o relato verbal e as leis da psicofísica são relevantes para se compreender o fenômeno da experiência dolorosa. Por meio de escalas unidimensionais e multidimensionais da dor é possível analisar os dados objetivos e subjetivos deste fenômeno, de forma a inferir o nível de consciência dos indivíduos (ALBINO FILHO, PEREIRA JÚNIOR, 2017).

Neste sentido, Coleman (2019) discute que a dor (tanto do ponto de vista objetivo quanto subjetivo) pode estar presente mesmo em situações de rebaixamento do nível de consciência. Argumenta que em situações de hipnose ou anestesia, a dor é evidenciada pelo aumento da pressão sanguínea e da frequência cardíaca e, por consequência, o aspecto subjetivo também está presente, embora, não necessariamente, consciente. Tal constatação, também corrobora com a unidade dos aspectos necessários à experiência consciente.

Por fim, argumenta-se que para avaliar a experiência consciente é importante verificar, para cada pessoa, o estabelecimento de correspondência confiáveis entre estados conscientes subjetivos e estados objetivos mensuráveis, sendo as escalas de avaliação do nível de consciência instrumentos importantes para esta finalidade.

\section{CONSIDERAÇÕES FINAIS}

O problema epistemológico de avaliar a experiência consciente por meio de sinais objetivos está presente na prática clínica na área da Saúde. O uso de escalas que parametrizam sinais objetivos da consciência (abertura ocular, 
respostas verbal, motora e dolorosa) é prática recorrente, o que justifica a necessidade de ampliar e aprofundar argumentos que embasem esta correspondência.

Argumenta-se que o 'problema difícil' e a 'lacuna explicativa' podem ser melhor abordados quando se analisa a experiência consciente com o Monismo Dual, deste modo se evitando uma postura cética, na prática médica, sobre a correspondência entre sinais mensuráveis e experiências subjetivas. Devemos levar em conta que cada aspecto objetivo precisa ser avaliado de forma individualizada e particularizada para a pessoa atendida. Como o avaliador interpreta os sinais na sua perspectiva de primeira pessoa, é ainda importante ressaltar que a intersubjetividade está presente em toda avaliação, tendo sido padronizada na comunidade científica por meio das escalas de avaliação do nível de consciência.

\section{REFERÊNCIAS}

ADAMS, Fred; AIZAWA, Ken. The bounds of cognition. Philosophical Psychology, v. 14, n. 1, p. 43-64, 2001.

ALBINO FILHO, M.A; PEREIRA JUNIOR, A. A experiência dolorosa. Complexitas Revista de Filosofia Temática, Belém, v. 2, n. 1, p. 3-19, jan./jun., 2017.

BORDINI, L. et al. Escalas de coma: uma revisão histórica. Arquivos de NeuroPsiquiatria, v. 68, n. 6, p. 930-937, 2010. Disponível em: <http://www.scielo.br/pdf/anp/v68n6/19.pdf>. Acesso em: 30 abr. 2017.

CASTRO, F. et al. Temperatura corporal, índice de Aldrete e Kroulik e alta do paciente da unidade de recuperação pós-anestésica. Revista da Escola de Enfermagem da USP, v. 46, n. 4, 2012. Disponível em: <http://www.scielo.br/pdf/reeusp/v46n4/13.pdf>. Acesso em: 17 jul. 2017.

CHALMERS, D. The conscious mind. Oxford: Oxford University Press, 1996.

CHOMSKY, N. Language and mind. 3 ed. New York: Cambridge University Press, 2006.

COLEMAN, S. Painfulness, suffering, and consciousness (Penultimate Draft). In: BAIN, D; BRADY, M; CORNS, J. (eds.). New York: Routledge, 2019.

DUARTE, L.; SARAIVA, R. Quando o índice bispectral (BIS) pode fornecer valores espúrios. Revista Brasileira de Terapia Intensiva, v. 59, n. 1, 2009. Disponível em: <http://www.scielo.br/pdf/rba/v59n1/13.pdf>. Acesso em: 8 fev. 2017.

EVERS, A. S; CROWDER, C, M. Anestésicos Gerais. In: GOODMAN. GILMAN (ed.). As bases farmacológicas da terapêutica. 10 ed. Rio de Janeiro: McGraw-Hill, 2005.

FAGUNDES, J. O. A. Sobre estados mentais relacionais e qualia: o totalmente subjetivo não pode ser conhecido. In: TOLEDO, G. L; GOUVEA, R. A. S; ALVES, M. A. S (org). Debates Contemporâneos em Filosofia da Mente. São Paulo: FiloCzar, 2018.

JOHN, E. R. et al. Source imaging of QEEG as a method to detect awareness in a person in vegetative state. Brain Injury, v. 25, n.4, p. 426-432, 2011. 
KANDEL, E. et al. Princípios de neurociências. 5 ed. Porto Alegre: AMGH, 2014.

LE DOUX, J. The emotional brain, fear, and the amigdala. Cellular and Molecular Neurobiology, v. 23, n. 4-5, p. 727-738, 2002.

MACHADO, A. Neuroanatomia funcional. 2 ed. São Paulo: Atheneu, 2007.

MENDES, C. et al. Escalas de Ramsay e Richmond são equivalentes para a avaliação do nível de sedação em pacientes gravemente enfermos. Revista Brasileira de Terapia Intensiva, v. 20, n. 4, 2008. Disponível em: <http://www.scielo.br/pdf/rbti/v20n4/v20n4a05.pdf>. Acesso em: 9 mar. 2017.

NITRINI, R.; LUIZ, A. B. A neurologia que todo médico deve saber. 2 ed. São Paulo: Editora Atheneu, 2010.

OLIVEIRA, D.; PEREIRA, C.; FREITAS, Z. Escalas para avaliação do nível de consciência em trauma cranioencefálico e sua relevância para a prática de enfermagem em neurocirurgia. Arquivos Brasileiros de Neurocirurgia, v. 33, n. 1, 2014. Disponível em: <http://files.bvs.br/upload/S/01035355/2014/v33n1/a4284.pdf>. Acesso em 15 jun. 2017.

PEDROZA, J. Monitorização neurológica. Rio de Janeiro: Sociedade de Anestesiologia do Estado do Rio de Janeiro, 2011.

PEREIRA JR, A. Triple-aspect monism: physiological, mental unconscious and conscious aspects of brain activity. Journal of Integrative Neuroscience, v. 13, n. 2, p. 201-227, 2014.

PEREIRA JR, A. The projective theory of consciousness: from neuroscience to philosophical psychology. Trans/Form/Ação, v. 41, n. especial, p. 199-232, 2018.

SCHURGER, A. et al. Reproducibility distinguishes conscious from nonconscious neural representations. Science, v. 327, p. 97-99, 2010. Disponível em: <https://www.ncbi.nlm.nih.gov/pubmed/19965385>. Acesso em: 7 dez. 2017.

VELMANS, M. Understanding consciousness. New York: Routledge, 2009.

Recebido em: 15-10-2018

Aceito para publicação em: 29-07-19 